\title{
Microwave-based biosensor for glucose detection
}

\begin{abstract}
In this project, microwave-based biosensor for glucose detection has been studied. The study is based on the dielectric properties changes at microwave frequency for glucose-enzyme reaction. Glucose interaction with glucose oxidase (GOD) produced gluconic acid and hydrogen peroxide. The reaction of the glucose solutions with an enzyme was carried out in 1:3 of glucose and enzyme respectively. The measurements were done using the Open Ended Coaxial Probe (OECP) coupled with computer controlled software automated network analyzer (ANA) with frequency range from $200 \mathrm{MHz}$ to $20 \mathrm{GHz}$ at room temperature (25 [PC). The differences of enzyme and glucose-enzyme reaction were calculated and plotted. In the microwave interaction with the glucose-enzyme reaction, ionic conduction and dipole molecules was detected at $0.99 \mathrm{GHz}$ and $16.44 \mathrm{GHz}$ respectively based on changes of dielectric loss factor.
\end{abstract}

Keyword: Microwave; Biosensor; Glucose 